\title{
The research on the visual obstacle-avoidance optimization in robots control
}

\author{
Tian Hongbin \\ Henan Polytechnic \\ Zhenzhou, 450046,China \\ 13938522103@139.com
}

\begin{abstract}
In order to increase the movement capability of the robotic visual system in three-dimension space, the paper designs an obstacle-avoidance algorithm based on robotic movement visual by effectively processing the visual information colleted by the robotics. This paper establishes a structural model of coordination control system. The obstacles can be effectively identified and avoided by the obstacleavoidance theory in the robotics coordination operation. The mathematical model of the obstacle-avoidance algorithm can predict the locations of the obstacles. The experiment proves the proposed algorithm can avoid the obstacles in threedimension space and the accuracy is very high.
\end{abstract}

Keywords-Moving robotics; visual location; obstacleavoidance.

\section{INTRODUCTION}

Intelligent robots are the machine which can sense, think and behave to acquire, process and identify information and it can automotive finish some complex operations [1]. Intelligent robots have very advanced brain in which the most important part is the central computer. This computer can control the behavior of the robotics according to the humans' operation. Intelligent robots have a variety of internal sensor and external information sensor, such as vision, hearing, touch, and smell sensors. Intelligent robots, which are as the automotive and information equipment, play an important role in the network world. Due to the diversity of the operation environment of the intelligent robots, the functions of the intelligent robots are various[2]. Thus the current research is hot to detect the static and dynamic obstacles and avoid the obstacles on time in the unknown environment. The $75 \%$ information of the human beings acquired is from the vision which can provide the overall environment information. The identifiable areas and corresponding obstacles, rather than the distinct exterior things should be identified when the moving robots navigate and avoid the obstacles[3]. Recently, with the development of computer visual image processing techniques and the advantage of visual system in signal detection area and data complete, visual sensors are widely applied in mobile robots navigation, obstacle identification. Due to the increasingly complete of the robots techniques and the application of mobile robots, the navigation techniques based on vision are key research issues. This technique has the advantage of information diversity, searching area wide and target information complete. The visual information contains large amount of data. It's necessary to attain complex algorithm and time-consuming computation to extract available information. How to ensure the real time and robust characteristics of the navigation system under the premise of finding correct path information is key research area[4,5].

\section{The Robots Visual Obstacle Avoidance ANALYSIS}

\section{A. 2.1 Robots distribution control system structure}

The robots control system contains central control and decentralized control. The decentralized control can operate simultaneously with high flexibility. However the decentralized control is hard to operate some tasks which need high coordinate level. The central control has better conformity and it can easily operate tasks with high coordinates requirements. But it has less flexibility and the computation has delay issue.

The robots controller in the robots system not only need to deal with the process paths design, control and coordinates issues, but also need to process large amount of exterior sensor information. Especially in the multi-robot coordinate system, the main task of the system is to coordinate, communicate and control The central control can properly distribute the tasks to ensure each robot has proper assignment, which can avoid the collision in the operation. The suitable assignment design can ensure the robots can operate independently to effectively finish the corresponding tasks.

\section{B. 2.2 Robots coordinate control system structure}

The scheme of the robots coordinate control system structure based on distribution control theory is as shown in figure 1

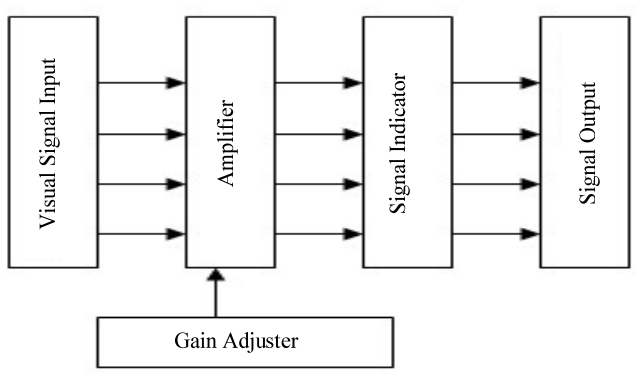

Figure 1. the scheme of the amplifier

There are two types of three-tier robots coordinate control structure. The first tier is computer control base 
station, the operation environment is that the frequency of CPU is $3 \mathrm{GHz}$, memory is $2 \mathrm{G}$, operation system is Windows XP, Motocom64 communication packages, robot off-line program and simulation environment. The paths without collision are analyzed by computer control base station and the simulation results are outputted to the middle tier to control the robots. The second tier contains ERCREA1400N-AA00, and power connection unit JZRCRNTU01-1. The functions of the second tier are to control signal communication, design work, and sensor control. The third tier is control level to control the whole system which contains support motors and actuators.

\section{The Visual ObStacle-Avoidance Model}

The moving robots themselves have some heights. Thus not only the obstacles in the moving trajectory of the base points can collide with the robots. Assuming the lateral dangerous distance Ds means the robot can pass from one side of the obstacle, it's the longest distance that the base point of the robots may collide with the obstacles. Ds can be determined by following equation.

$$
D_{\mathrm{s}}=k_{\mathrm{s}} w_{\mathrm{ks}}>1
$$

The effect range of the point obstacle $\mathrm{P}$ with known position in the space is considered. If the shortest distance of some angle between the radial radius and $\mathrm{P}$ is not large than Ds, the collision may happen when keeps moving among the direction. In figure 2, the angle between the two lines may be affected by the obstacles with the probability of danger. There are some resistances in the angle area for P.

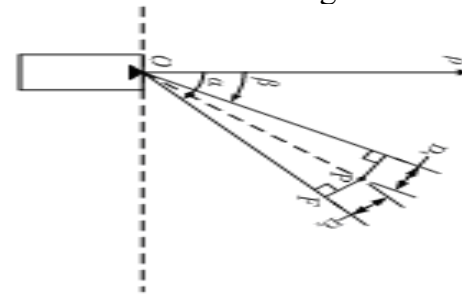

Figure 2. The effect rang of the obstacle points

Assuming the polar coordinator of $\mathrm{P}$ in the coordinate system is $(\rho \mathrm{P}, \theta \mathrm{P})$, the resistance rang $[\alpha \mathrm{P}, \beta \mathrm{P}]$ of $\mathrm{P}$ in the angle area can be attained by simple triangular relationship.

$$
\left\{\begin{array}{l}
\alpha_{P}=\theta_{P}-\arcsin \frac{D_{\mathrm{s}}}{\rho_{P}} \\
\beta_{P}=\theta_{P}+\arcsin \frac{D_{\mathrm{s}}}{\rho_{P}}
\end{array}\right.
$$

The above is the discussion of ideal situation, without considering the barrier of the mobile robots. Actually the mobile robots can't move along the line due to the barrier of the mobile instantaneous direction when the target direction is out of barrier. The revised resistance angle range $\left[\alpha_{P}^{\prime}, \beta_{P}^{\prime}\right]_{\text {due to } \mathrm{P} \text { is: }}$

$$
\begin{aligned}
& \alpha_{P}^{\prime}= \begin{cases}\theta_{P}-\arcsin \frac{D_{\mathrm{s}}}{\rho_{P}}, & \left|D_{1 P}-\rho_{1}\right|>D_{\mathrm{s}} \\
-\frac{\pi}{2}, & \left|D_{1 P}-\rho_{1}\right| \leq D_{\mathrm{s}}\end{cases} \\
& \beta_{P}^{\prime}= \begin{cases}\theta_{P}+\arcsin \frac{D_{\mathrm{s}}}{\rho_{P}}, & \left|D_{2 P}-\rho_{2}\right|>D_{\mathrm{s}} \\
\frac{\pi}{2}, & \left|D_{2 P}-\rho_{2}\right| \leq D_{\mathrm{s}}\end{cases}
\end{aligned}
$$

Assuming the resistance is inversely proportional with the expected path length. When the length of the expect path is approaching 0 , the resistance is approaching infinity; when the length is approaching infinity, the resistance is approaching 0 . In figure 2 , the obstacle $\mathrm{P}$ is in the area of the angle range $[\alpha, \beta]$. The longest expect path of the mobile robots from current time to the collision time is $\mathrm{OP}$, the length is $\rho \mathrm{P}$, expected shortest path is $\mathrm{OF}$ and the length is ( $\rho$ P $2-D s) 1 / 2$. In order to simplify computation, assuming the obstacle can generate the same resistance in the effect angle area, the shortest length of the collision path can be used to compute the resistance in the angle area. The resistance generated by the obstacle is expressed as follows.

$$
F_{P}(\theta)= \begin{cases}\frac{1}{\sqrt{\rho_{P}^{2}-D_{\mathrm{s}}^{2}}}, & \alpha_{P}^{\prime} \leq \theta \leq \beta_{P}^{\prime} \\ 0, & \theta<\alpha_{P}^{\prime} \text { 或 } \theta>\beta_{P}^{\prime}\end{cases}
$$

For some angle $\theta$, the total resistance is regarded as the resistance generated by all the obstacles in the angle and as the largest value. The total resistance function is:

$$
F_{\mathrm{r}}(\theta)=\max \left(F_{P_{1}}(\theta), F_{P_{2}}(\theta), \cdots, F_{P_{n}}(\theta), F_{\mathrm{b}}(\theta)\right)
$$

In the above equations, $\mathrm{P} 1 \sim \mathrm{Pn}$ are the obstacles in the area. The collision will definitely happen if not avoid.

\section{Robot CONTROL AND SIMULATION EXPERIMENT}

In order to verify the validity of the proposed visual obstacle avoidance algorithm in robot control, it's necessary for the simulation experiment. The experiment designs the robots work path and preset some collision area based on central and distribute three-tier coordinate control system. The experimental sample is pioneer3-DX with 18 visual sensors which can collect obstacle and target information from different directions. The control system of the robot contains three modules-data communication, target searching, and obstacle avoidance. During the operation of the experiment, the robot can priority avoid the obstacles due to the priority of the obstacles and target searching modules and direct to corresponding visual target. The details are described as figure 3 . 


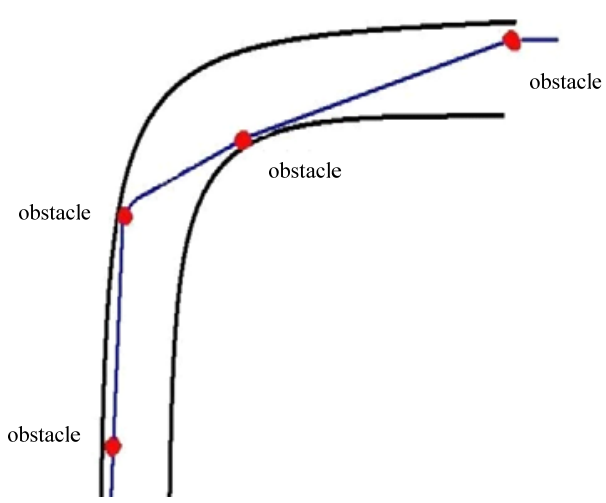

Figure 3. mobile robot simulation experiment results

The detailed statistical results are described as follows.

TABLE I. EXPERIMENT DATA ANALYSIS

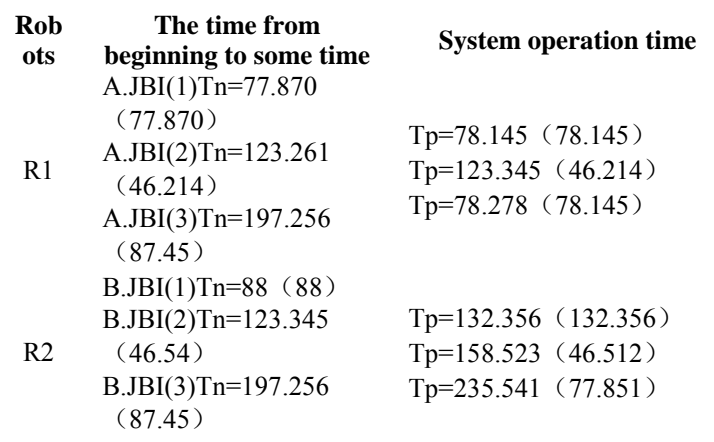

From table 1, the average independent operation time of the obstacle-avoidance is $172.13 \mathrm{~s}$ the total system operation time is $259.768 \mathrm{~s}$. The waiting time in point 2 is $55.294 \mathrm{~s}$. If there are corresopingding collision areas, the normal control method is to make the robot stop or control different robots to work independently. The proposed algorithm visual obstacle-avoidance optimization method can greatly increase the efficiency of the later control, which illustrates the proposed method can fulfill the obstacle avoidance in the three-dimension space and with high accuracy.

\section{CONCLUSIONS}

This paper designs an obstacle-avoidance algorithm based on robotic movement visual by effectively processing the visual information colleted by the robotics. This paper establishes a structural model of coordination control system. The obstacles can be effectively identified and avoided by the obstacle-avoidance theory in the robotics coordination operation. The mathematical model of the obstacleavoidance algorithm can predict the locations of the obstacles. The experiment proves the proposed algorithm can avoid the obstacles in three-dimension space and the accuracy is very high.

\section{REFERENCES}

[1] Yam suchi. Brian Decentralized coordination for multirobot exploration[J], Robotics and Autonomous Systemss, 1999,29(1):543 544.

[2] A sama $\mathrm{H}$, et al. Design of an autonomous and distributed robot system[J]. ACTRESS[A] Proc IEEE/RSJ 2000:283 286.

[3] A.Webb.Statistical Pattern Recognition Arnokd Publishers,London, 1999.

[4] M.A.T..Figueirdo,A.K.Jain.Unsupervised learning of finite mixture models.IEEE Transactions on Pattern Analysis and Machine Intelligence, 2002, 24(3):381 396.

[5] H.X.Wang,B.Luo,Q.B.Zhang,S.Wi.Estimation for the number of components in a mixture model using stepwise split-and-merge EM algorithm.Patrrern Recognition Letters,25(16):1799-1809,December 2004. 